\title{
The ubiquitin system: from cell signalling to disease biology and new therapeutic opportunities
}

\author{
Rune Busk Damgaard (i) ${ }^{1}$
}

Received: 25 November 2020 / Accepted: 27 November 2020 / Published online: 14 January 2021

(c) The Author(s), under exclusive licence to ADMC Associazione Differenziamento e Morte Cellulare 2021

Forty years ago, ubiquitin was first discovered as a posttranslational modification earmarking proteins for degradation [1]. This seminal, biochemical work laid the foundation for countless discoveries that revolutionised our understanding of cell biology and physiology. Today, we know that ubiquitin regulates virtually all aspects of eukaryotic biology. All eukaryotes - from yeast to humans-express the enzymatic machinery to covalently modify substrate proteins with ubiquitin, a 76 amino-acid residue protein, in a process termed ubiquitination (Fig. 1A). Ubiquitination is a three-step enzymatic cascade involving E1, E2, and E3 enzymes that results in the transfer of ubiquitin, via its Cterminal glycine, onto the $\varepsilon$-amino group of a lysine residue on the substrate [2]. The reverse reaction-removal of ubiquitin modifications from substrates-is performed by deubiquitinases (DUBs) [3]. Ubiquitination has emerged as one of the most pervasive post-translational protein modifications. Tens-of-thousands of ubiquitination sites on thousands of proteins have been identified, indicating that most cellular proteins will experience ubiquitination at some point in their lifetime.

Ubiquitination starts by the transfer of a single ubiquitin to a substrate lysine residue. These monoubiquitination events are abundant in cells and serve many purposes. But a hallmark of ubiquitin is that it can be assembled into polymeric chains through ubiquitination of any of the seven internal lysine residues (Lys6, Lys11, Lys27, Lys29, Lys33, Lys48, and Lys63) or the $\alpha$-amino group of the N-terminal methionine (Met1) within ubiquitin itself. This results in the assembly of eight types of homotypic polyubiquitin chains and a broad range of heterotypic, or mixed, and

Rune Busk Damgaard

rudam@dtu.dk

1 Department of Biotechnology and Biomedicine, Technical University of Denmark, Søltofts Plads, 2800 Kgs. Lyngby, Denmark branched polyubiquitin chains, collectively referred to as the 'ubiquitin code' [2] (Fig. 1B). Recent discoveries on the assembly, regulation, and decoding of the ubiquitin code have revealed that the different polyubiquitin linkages are structurally and functionally distinct signals that serve different cellular purposes. Even heterotypic and branched ubiquitin chains may be considered discrete cellular signals $[2,4]$. The detailed biochemical characterisation of linkage-specific ubiquitin ligases or DUBs that assemble or disassemble either one or a discrete set of ubiquitin modifications has transformed the field. Together with technological advances in quantitative mass spectrometry-based proteomics methods and improved affinity reagents, it has led to the development of new tools and workflows to study signalling by specific types of ubiquitin modifications [2-4]. With these new tools and insights into the 'ciphers' of the 'code', we are getting closer to decrypting how these complex ubiquitin signals regulate cell biology.

Ubiquitin modifications control a plethora of vital cellular processes through proteolytic and nonproteolytic mechanisms, including proteasomal degradation and proteostasis, selective autophagy, cell signalling cascades such as those for NF- $\mathrm{KB}$ activation, protein trafficking, DNA repair and genome integrity, cell cycle control, and programmed cell death (Fig. 1C). It is therefore not surprising that dysregulation of ubiquitination can have detrimental consequences for cells. It may for instance lead to deactivation or aberrant activation of signalling pathways, accumulation of damaged or misfolded proteins, or mislocalisation or mistrafficking of proteins away from their cognate compartment; each of which can severely impede or damage cell function. Thus, similar to kinases, dysregulation of ubiquitin-dependent signalling leads to a variety of diseases such as congenital developmental defects, cancer, neurodegenerative diseases, and immune disorders (Fig. 1D). This makes the enzymes of the ubiquitin system attractive drug targets $[5,6]$.

The thirteen reviews that follow this introduction discuss some of the most recent and interesting advances in our 
Fig. 1 Ubiquitin signalling in cell biology and disease.

A Schematic of the

ubiquitination and

deubiquitination reactions.

Ubiquitination is a sequential

cascade of enzymatic reactions.

First, ubiquitin (Ub) is attached

to the E1 Ub-activating enzyme in an ATP-dependent manner before it is transferred to an E2 Ub-conjugating enzyme. Subsequently, the $\mathrm{Ub}$ is transferred from the $\mathrm{Ub} \sim \mathrm{E} 2$ conjugate to a lysine residue in a substrate either directly from the E2, mediated by a RING E3 Ub ligase, or first transferred to a HECT/RBR E3 Ub ligase before being transferred to the substrate. Deubiquitination is performed by deubiquitinases (DUBs) that remove ubiquitin modifications from substrates.

B Schematic representation and examples of some of the ubiquitin modifications in the 'ubiquitin code'. C Examples of cellular functions that are regulated by ubiquitination. D Dysfunction or dysregulation of ubiquitination is associated with development of a range of pathologies and human diseases. Recent advances in the understanding of ubiquitin biology at the cellular and molecular level may offer new therapeutic opportunities and strategies for drug development to treat human diseases. HECT Homologous to the E6-AP Carboxyl Terminus, RING really interesting new gene, RBR RING-in-between-RING.
A

Ubiquitination and deubiquitination

Ub
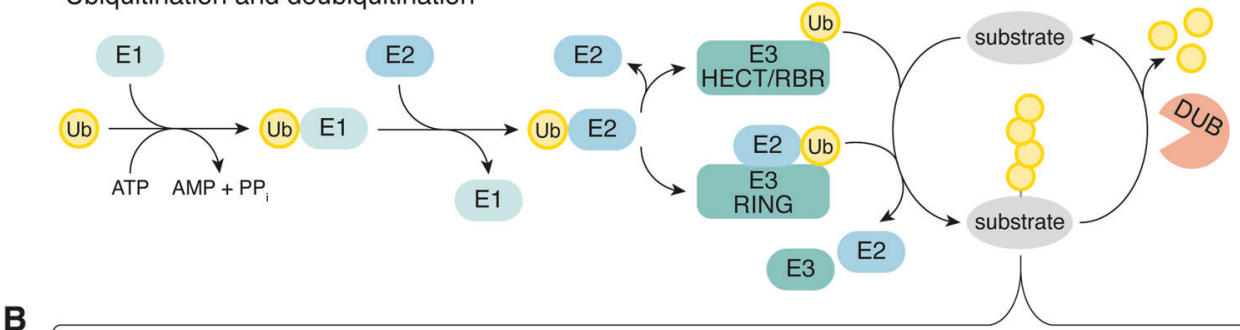

The ubiquitin code

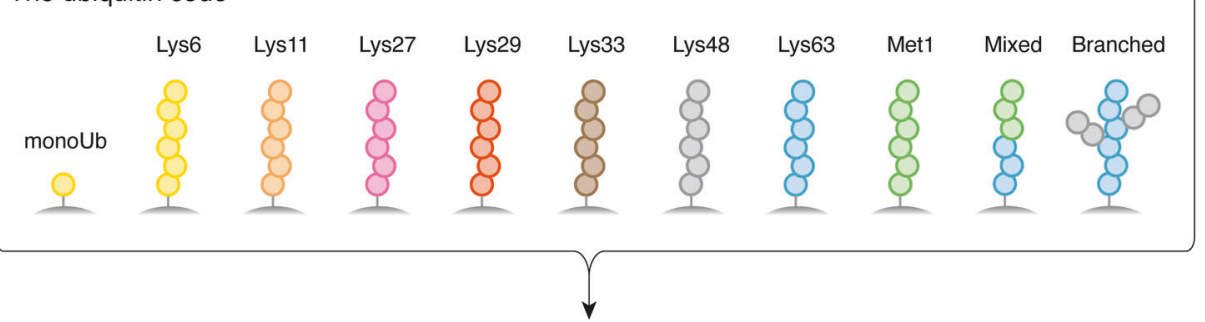

C

Cellular functions of ubiquitination

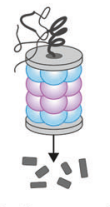

Proteasomal degradation

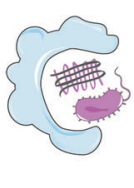

Selective autophagy

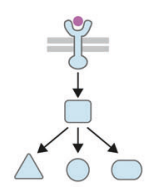

Cell signalling, e.g. NF-KB

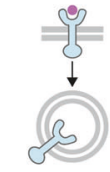
Endocytosis and

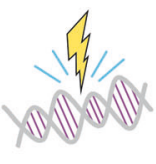

DNA damage response
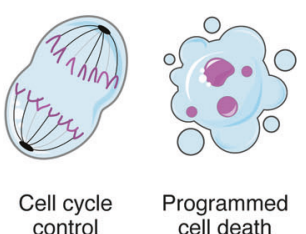

control
Programmed cell death

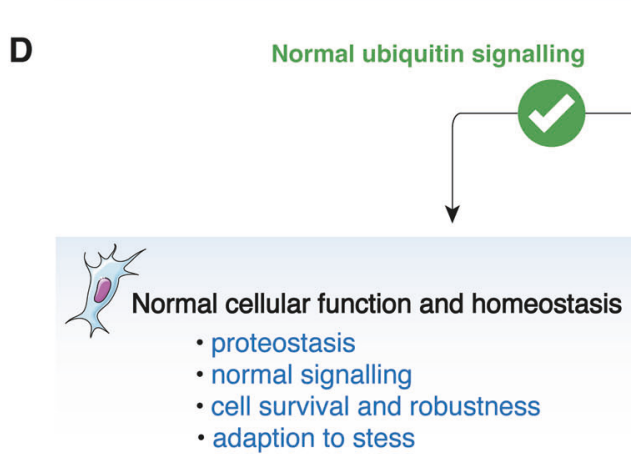

Dysfunctional ubiquitin signalling

New therapeutic opportunities in the ubiquitin system?

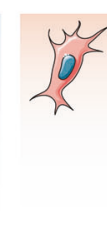

Cellular dysfunction and disease

- developmental diseases

- cancer

- immune disorders

- neurogenerative diseases

- metabolic disorders

$\cdot$ ageing understanding of the role and regulation of ubiquitindependent signalling processes in disease biology and highlight new therapeutic opportunities within the ubiquitin system. Shah and Kumar [7] review emerging regulatory principles in ubiquitin code assembly, namely the control of HECT E3 ubiquitin ligases by adaptor proteins and the regulatory effect these accessory molecules have on physiological outcomes of signalling. Three additional reviews discuss the function and regulation of a single-but crucial-cipher of the ubiquitin code: Met1-linked ubiquitin chains. Fiil and Gyrd-Hansen [8] focus on the molecular concepts by which Met1-linked ubiquitin chains control inflammatory signalling pathways, NF- $\kappa \mathrm{B}$ activation, and processes during infection. Jahan et al. [9] review the variety of cellular functions controlled by Met1-linked ubiquitin chains and the diseases linked to dysregulation of this chain type, including immune disorders and cancer, and highlight emerging cellular roles of Met1-linked ubiquitin chains such as regulation of protein quality control and metabolism. Finally, Weinelt and van Wijk [10] examine the termination of Met1-linked ubiquitin signalling by the catalysis-dependent and -independent functions of the Met1-linkage-specific DUB OTULIN, particularly in relation to immune signalling and cell fate decisions.

In a review closely related to the theme of immune signalling, Cockram et al. [11] discuss the wide 
range of ubiquitin-mediated signalling mechanisms that control inflammatory cell death pathways, necroptosis and pyroptosis, and the roles these processes play in cancer development. Three more reviews explore additional important roles of ubiquitin signalling in cancer. Dang et al. [12] discuss how ubiquitination, particularly the two E3 ubiquitin ligases anaphasepromoting complex/cyclosome (APC/C) and the Skp1Cul1-F-box 8 (SCF) complex, regulate cell cycle progression and how APC/C and SCF complex dysregulation results in uncontrolled cell proliferation and tumorigenesis. Louis et al. [13] review the current knowledge of the function of the tumour suppressor DUB BAP1, including its roles in cell proliferation, differentiation, metabolism, and cell death, and discuss how inactivation of this DUB might cause cancer. Lastly, Humphreys et al. [14] focus on the role of E3 ubiquitin ligases in the development and progression of glioblastoma, an aggressive form of brain cancer, and discuss how the ubiquitin system may be exploited in the future for novel therapeutic interventions.

In the remaining reviews of this collection, we follow ubiquitin 'from cradle to grave', in a broad sense. Basar et al. [15] review the emerging role of DUBs in control of gene expression of chromatin regulation during embryonic development and discuss how dysregulation of DUBs can cause developmental disorders. In extension of this, Ottens et al. [16] explore the coordinated regulation of proteostasis and metabolism that is crucial for preventing ageing and ageing-related diseases such as neurodegeneration. Neurodegenerative diseases, often caused by accumulation of toxic protein aggregates that escape degradation, are a growing burden worldwide. Three reviews in this collection explores the current knowledge of ubiquitindependent proteolysis and signalling in neurodegeneratve diseases. Le Guerroué and Youle [17] focus on the role of ubiquitin-dependent degradation of misfolded proteins and aggregates, such as $\alpha$-synuclein and amyloid- $\beta$, associated with neurodegenerative diseases and discuss possible reasons why the ubiquitin system fails to eliminate aggregates. Schmidt et al. [18] review the role of ubiquitindependent signals in mitochondrial homeostasis, membrane receptor trafficking, neuroinflammation, and neuronal cell death in the development of neurodegenerative diseases and provide a thorough analysis of the emerging therapeutic opportunities found within the ubiquitin system for the treatment of neurodegeneration. Finally, Ziv and Ciechanover [19] discuss new results showing that site-specific ubiquitination of aggregate-prone Huntingtin, the causative agent in Huntington's disease, may alleviate the neuropathology in a surprising way: namely by enhancing-rather than preventing-the formation of large protein aggregates.
Collectively, these outstanding reviews bring the readership of Cell Death \& Differentiation a comprehensive overview of fascinating and emerging concepts of ubiquitinmediated processes in disease development. Time will tell if the promising therapeutic opportunities, which targeting ubiquitin-dependent processes provides, will bring the desired and much needed outcomes in the form of new treatments for human diseases.

Acknowledgements R.B.D. is supported by the Technical University of Denmark and the Novo Nordisk Foundation [NNF19OC0054248]. The figure was prepared using Servier Medical Art (https://smart. servier.com) with assistance from Camilla Reiter Elbæk.

\section{Compliance with ethical standards}

Conflict of interest The author declares that he has no conflict of interest.

Publisher's note Springer Nature remains neutral with regard to jurisdictional claims in published maps and institutional affiliations.

\section{References}

1. Ciechanover A. The unravelling of the ubiquitin system. Nat Rev Mol Cell Bio. 2015;16:322-4.

2. Komander D, Rape M. The ubiquitin code. Annu Rev Biochem. 2012;81:203-29.

3. Clague MJ, Urbé S, Komander D. Breaking the chains: deubiquitylating enzyme specificity begets function. Nat Rev Mol Cell Biol. 2019;20:338-52.

4. Swatek KN, Komander D. Ubiquitin modifications. Cell Res. 2016;26:399-422.

5. Huang $X$, Dixit VM. Drugging the undruggables: exploring the ubiquitin system for drug development. Cell Res. 2016;26: 484-98.

6. Harrigan JA, Jacq X, Martin NM, Jackson SP. Deubiquitylating enzymes and drug discovery: emerging opportunities. Nat Rev Drug Discov. 2018;17:57-78.

7. Shah SS, Kumar S. Adaptors as the regulators of HECT ubiquitin ligases. Cell Death Differ. https://doi.org/10.1038/s41418-02000707-6.

8. Fiil BK, Gyrd-Hansen M. The Met1-linked ubiquitin machinery in inflammation and infection. Cell Death Differ. https://doi.org/10. 1038/s41418-020-00702-x.

9. Jahan AS, Elbæk CR, Damgaard RB. Met1-linked ubiquitin signalling in health and disease: inflammation, immunity, cancer, and beyond. Cell Death Differ. https://doi.org/10.1038/s41418-02000676-w.

10. Weinelt N, van Wijk SJL. Ubiquitin-dependent and -independent 1 functions of OTULIN in cell fate control and beyond. Cell Death Differ. https://doi.org/10.1038/s41418-020-00675-x.

11. Cockram PE, Kist M, Prakash S, Chen S-H, Shemorry A, Vidal S, et al. Ubiquitination in the regulation of inflammatory cell death and cancer. Cell Death Differ. https://doi.org/10.1038/s41418020-00708-5.

12. Dang F, Nie L, Wei W. Ubiquitin signaling in cell cycle control and tumorigenesis. Cell Death Differ. 2020;1-12. https://doi.org/ 10.1038/s41418-020-00648-0.

13. Louis M, Oumaima A, Estavoyer B, Larrivée B, Labrecque N, Nijnik A, et al. Roles and mechanisms of BAP1 deubiquitinase in 
tumor suppression. Cell Death Differ. https://doi.org/10.1038/ s41418-020-00709-4.

14. Humphreys LM, Chen Z, Fouad S, Smith P, D'Angiolella V. The role of E3 ubiquitin ligases in the development and progression of Glioblastoma. Cell Death Differ. https://doi.org/10.1038/s41418020-00696-6.

15. Basar MA, Beck DB, Werner A. Deubiquitylases in embryonic ubiquitin signaling and developmental diseases. Cell Death Differ. https://doi.org/10.1038/s41418-020-00697-5.

16. Ottens F, Franz A, Hoppe T. Build-UPS and break-downs: metabolism impacts on proteostasis and aging. Cell Death Differ. https://doi.org/10.1038/s41418-020-00682-y.
17. Guerroué FL, Youle RJ. Ubiquitin signaling in neurodegenerative diseases: an autophagy and proteasome perspective. Cell Death Differ. 2020;1-16. https://doi.org/10.1038/s41418-020-00667-x.

18. Schmidt MF, Gan ZY, Komander D, Dewson G. Ubiquitin signalling in neurodegeneration: mechanisms and 2 therapeutic opportunities. Cell Death Differ. https://doi.org/10.1038/s41418020-00706-7.

19. Ziv NE, Ciechanover A. A possible non-proteolytic role of ubiquitin conjugation in alleviating the pathology of Huntingtin's aggregation. Cell Death Differ. 2020;1-4. https://doi.org/10.1038/ s41418-020-00617-7. 